\title{
Digitale Verhaltensdaten und Methoden der Computational Social Science in der politischen Kommunikationsforschung
}

Sebastian Stier und Andreas Jungherr

\section{Einleitung}

Durch die Digitalisierung unterliegt die politische Kommunikation einem fundamentalen Wandel. Bürger ${ }^{1}$ in etablierten Demokratien und darüber hinaus greifen zunehmend über Websites oder Smartphone Apps auf Nachrichten zu und beteiligen sich über soziale Netzwerkseiten (SNS) wie Facebook oder Twitter aktiv am politischen Diskurs (Newman et al. 2018). Die politische Kommunikationsforschung greift zur Analyse dieser Trends vermehrt auf digitale Verhaltensdaten und computergestützte Methoden zurück; Ansätze, die häufig unter dem interdisziplinären Forschungsparadigma der Computational Social Science (CSS) gefasst werden (Lazer et al. 2009).

Digitale Verhaltensdaten (englisch »digital trace data«), können definiert werden als "records of activity (trace data) undertaken through an online information system (thus, digital)« (Howison et al. 2011, 769). Diese Spuren menschlicher Aktivität, z.B. in sozialen Netzwerken oder über Smartphones verzeichnet, eröffnen einen bislang nicht möglichen, direkten Einblick in das Verhalten von Bürgern und politischen Eliten und sind dabei nicht-intrusiv, d.h. die Beobachtungsobjekte werden durch Interventionen des Forschers nicht beeinflusst. Die resultierenden Daten sind multimodal (Text, Videos), temporal hochauflösend, dank präziser Zeitstempel, und legen so je nach Anwendungsgebiet Spuren der politischen Kommunikation und Informationsrezeption von Millionen Bürgern offen. Das vorliegende Kapitel gibt einen kurzen Abriss über relevante Forschung im Feld, reflektiert diese kritisch und skizziert schließlich eine zukünftige Forschungsagenda, die die theoretischen Stärken der Sozialwissenschaften und den methodischen Innovationsgehalt der CSS gewinnbringend zusammenführt.

1 Aus Gründen der besseren Lesbarkeit wird das generische Maskulinum genutzt. 


\section{Forschungsstand}

SNS wie Facebook und Twitter gewinnen zunehmend an Relevanz für die Rezeption politischer Informationen sowie Meinungsbildungs- und Partizipationsprozesse. Sie wurden deshalb schnell von der politischen Kommunikationsforschung in den Blick genommen. Im Folgenden sollen zentrale Themen und methodische Trends umrissen werden. ${ }^{2}$

\section{Wahlkampf- und Parteienforschung}

Digitale Verhaltensdaten, insbesondere Twitter-Daten, sind längst ein etabliertes Mittel zur Erforschung von Wahlkämpfen und ihren Dynamiken (Jungherr 2016; siehe auch Borucki 2019 in diesem Band). Dabei ist zwischen publikums- und elitenzentrierten Forschungsdesigns zu unterscheiden. Publikumszentrierte Studien interessieren sich für die politische Kommunikation von Bürgern, indem Tweets mit kampagnenbezogenen Schlagworten oder Hashtags wie z.B. \#btw17 oder \#MAGA erfasst werden. Diese Forschung konzentrierte sich häufig auf Fokuspunkte wie das "Second Screening « während TV-Debatten (Freelon/Karpf 2015; Jungherr 2014; Trilling 2015; Vaccari et al. 2015a), einflussreiche Nutzer (Dubois/Gaffney 2014; Jungherr 2015; Jürgens et al. 2011) und die Identifikation zentraler politischer Themen (Jungherr 2015; Trilling 2015). Der Großteil der Wahlkampfforschung konzentriert sich auf Twitter, da im Gegensatz zum anderen sozialen Netzwerk mit großer politischer Relevanz, Facebook, die meisten Nutzerbeiträge öffentlich sind. Ausnahmen hierzu bilden jedoch Studien, die Publikumsreaktionen auf FacebookSeiten politischer Parteien oder Spitzenkandidaten untersuchen (Arzheimer 2015; Stier et al. 2017; siehe auch Fitzpatrick 2019 in diesem Band).

Publikumszentrierte Analysen demonstrieren unabhängig von ihren spezifischen Fragestellungen das beträchtliche Forschungspotential digitaler Spurendaten, die den politischen Diskurs in (Teil-)Öffentlichkeiten dokumentieren. So erhalten wir Einblick in die Verteilung und Dynamik öffentlicher Aufmerksamkeit (Freelon/Karpf 2015; Jungherr et al. 2017), die öffentliche Interpretation und Verhandlung von Wahlkampfereignissen (Trilling 2015; Vaccari et al. 2015a; Zhang et al. 2019) oder das Verhalten von Diskursteilnehmern in unterschiedlichen politischen Kommunikationsumgebungen (An et al. 2019; Freelon und Karpf 2015). Dadurch verspricht die CSS, etablierte Forschungszweige der politischen Kommunikationsforschung zu bereichern.

2 Unsere Auswahl ist nicht erschöpfend. Es gibt weitere Themen- und Methodenschwerpunkte wie z.B. agentenbasierte Modelle, deren Nutzung in der politischen Kommunikationsforschung allerdings noch am Anfang steht. 
Einige publikumszentrierte Studien versuchten sich zudem an der Vorhersage von Wahlergebnissen auf Basis von Social Media-Daten (z.B. Tumasjan et al. 2011). Diese Studien entpuppten sich allerdings als wenig robust und konzeptionell unterfundiert. Da das Zielkonstrukt Wahlverhalten nicht über einen plausiblen Mechanismus mit den Aktivitäten einer kleinen Zahl an politisch aktiven Twitterern in Zusammenhang gesetzt werden kann, sind die veröffentlichten Erfolgsmeldungen nicht mehr als Scheinkorrelationen (Jungherr et al. 2012, 2017). Es gibt allerdings auch Ansätze, die Online- und Offline-Sphären zueinander in Bezug setzen, ohne diese falschen Inferenzschlüsse zu ziehen, indem Verhaltensdaten in SNS mit Umfrage- oder Mediendaten in Bezug gesetzt werden (Jungherr 2014; Jungherr et al. 2016; Neuman et al. 2014; Posegga/Jungherr 2019; Stier et al. 2018a; Wells et al. 2016).

Elitenzentrierte Ansätze fokussieren auf eine wohl definierte Gruppe an Accounts von Interesse, meist Kandidaten, Parteien oder Journalisten. Studien konzentrierten sich auf die Adaption einer Plattform durch Politiker (Quinlan et al. 2017; Vergeer et al. 2013), die parteipolitische Zusammensetzung von Kandidatennetzwerken bzw. Fragmentierungstendenzen entlang von Parteizugehörigkeit (Conover et al. 2011; Lietz et al. 2014) und die Resonanz von Eliten beim Publikum anhand von Interaktionsmetriken, wie die Zahl der Likes oder Retweets (Nielsen/Vaccari 2013; Yang/Kim 2017). Eine wachsende Zahl an Studien untersucht zudem, inwiefern Politiker und Parteien in sozialen Medien eine populistische Rhetorik bedienen (Ernst et al. 2017) oder populistische Parteien und Bewegungen in ihren Themenschwerpunkten von etablierten Parteien abweichen (Stier et al. 2017). Die Ergebnisse elitenzentrierter Ansätze demonstrieren, dass bekannte Variablen wie die Ressourcenstärke, der Bekanntheitsgrad und die Parteizugehörigkeit weiterhin zentrale Bestimmungsfaktoren des Verhaltens und des Erfolgs von Parteien oder Politikern in SNS sind.

Insgesamt weist diese Literatur aber auch einige Defizite auf. So basiert die Fallauswahl häufig auf Ad-hoc-Entscheidungen wie z.B. wenigen ausgewählten (Partei-)Accounts oder Schlagwörtern während einer Kampagne. Mögliche Defizite dieser Sampling-Strategien werden kaum thematisiert oder auf ihre Robustheit hin überprüft. Zumeist verharren die Analysemethoden im Bereich simpel messbarer Metriken wie Likes, Retweets oder Schlagwortnennungen anstatt das volle methodische Portfolio der CSS, z.B. (semi-)automatisierte Inhaltsanalysen auszuschöpfen.

\section{Digitale Öffentlichkeit und Connective Action}

Die mediale Agenda in etablierten Demokratien wird mittlerweile stark durch die politische Kommunikation in sozialen Medien beeinflusst (Chadwick 2013; Neuman et al. 2014; Wells et al. 2016). Insbesondere auf Twitter interagieren Journalis- 
ten, Politiker und interessierte politische Bürger miteinander und interpretieren das politische Geschehen in Echtzeit. Dieser Diskurs erreicht dann über Rückkopplungsschleifen im sogenannten »hybriden Mediensystem« ein größeres Publikum (Chadwick 2013). Aktivisten und einfache Bürger, die sich in spezifischen Themennetzwerken engagieren (z.B. netz- oder umweltpolitischen Themen), nehmen im politischen Meinungsbildungsprozess zumindest zeitweise eine zentralere Rolle ein als im analogen, von Journalisten und Politikern dominierten Mediensystem (Bennett et al. 2018; Entman/Usher 2018). Einige Studien verfolgten die verwandte Frage, inwiefern die journalistische Praxis auf Twitter etablierten Normen entspricht oder davon abweicht (Nuernbergk 2016).

Insbesondere Protestbewegungen während des Arabischen Frühlings und in westlichen Demokratien wie Occupy Wall Street oder die spanischen Indignados haben demonstriert, dass bislang marginalisierte Akteure durch digitale Kommunikations- und Vernetzungsmöglichkeiten begünstigt werden können. Netzwerkanalytische Verfahren zeigen, dass durch die "connective action« (Bennett/Segerberg 2013) in SNS auch weniger politisch Involvierte an der Peripherie von Kommunikationsnetzwerken zumindest zu virtuellen Teilen von Protestbewegungen werden können (Barberá et al. 2015b; Bennett et al. 2018; Steinert-Threlkeld 2017). Allerdings zeigt sich, dass trotz des Optimismus hinsichtlich einer Egalisierung politischer Kommunikation die etablierten Gatekeeper wie Massenmedien, Politiker und organisierte und ressourcenstarke Interessen weiterhin zumindest bei hinreichender eigener Aktivität eine zentrale Rolle in Online-Diskursen einnehmen (Stier et al. 2018b).

\section{Qualität politischer Informationen und versteckte Beeinflussung digitaler Öffentlichkeiten}

Mit der wachsenden politischen Relevanz digitaler Medien legt die Wissenschaft ein besonderes Augenmerk auf potentiell demokratieschädigende Aspekte digitaler Vernetzung. Dazu gehört die Furcht vor einer abnehmenden Qualität politischer Informationen, die sich in der öffentlichen Debatte am problematischen $\mathrm{Be}$ griff »Fake News« manifestiert, sowie versteckte Informationskampagnen, um im Schutze der Anonymität des Internets demokratische Öffentlichkeiten zu manipulieren.

Wissenschaftler sind vom Begriff »Fake News« weitgehend abgerückt oder bemühen sich zumindest um eine präzisere Konzeptualisierung. Einige Arbeiten unterscheiden zwischen Desinformationen, falschen Informationen, die intentional verbreitetet werden, und Missinformationen, falschen Informationen, die ohne größeres strategisches Ziel von individuellen Nutzern weitergeleitet werden, z.B. Gerüchte in sozialen Medien nach Breaking News-Events oder zur Flüchtlingskriminalität (Wardle/Derakhshan 2017, 20). Gleichwohl wurden noch keine Anstren- 
gungen unternommen, den Charakter, die Verbreitung und Wirkung dieser beiden »information disorders« (Wardle/Derakhshan 2017) vergleichend und in größerem Maßstab zu untersuchen.

Die aktuellen Forschungsergebnisse zur Bedeutung von Des- oder Missinformationen in SNS sind dementsprechend fragmentiert und widersprüchlich. Zwar waren z.B. viele der meistgelesenen Nachrichtenartikel auf Facebook im US-Wahlkampf 2016 einseitig politisch geprägt, irreführend oder sogar eindeutig falsch (Allcott/Gentzkow 2017). Laut einer Studie verbreiten sich Falschinformationen auf Twitter schneller als von Fact-Checkern als wahr klassifizierte Nachrichten (Vosoughi et al. 2018). Andererseits zeigen Studien aus dem USKontext, die digitale Verhaltensdaten mit Daten auf individueller Ebene verlinken, dass der Konsum von Des- und Missinformationen im Vergleich zu etablierten Nachrichtenquellen marginal ist (Grinberg et al. 2019; Guess et al. 2018). Außer einigen wenigen experimentellen Studien (z.B. Pennycook et al. 2018) ist zudem viel zu wenig zur Wirkung solcher Nachrichten bekannt. Es verbleiben zentrale Fragen, z.B. ob Bürger Des- und Missinformationen tatsächlich Glauben schenken und ob diese politische Einstellungen beeinflussen können. Wie schwerwiegend die Beeinflussung der US-Präsidentschaftswahl 2016 durch die Agenten der russischen Troll-Fabrik Internet Research Agency (IRA) beispielsweise ausfiel, ist allein anhand der Verbreitungszahlen ihrer Tweets und Facebook-Posts nicht bestimmbar. $^{3}$

Die oben beschriebenen methodischen Unsicherheiten wiegen noch schwerer im Falle der viel diskutierten Social Bots, computergestützten (semi-)automatisierten Accounts, die (politische) Debatten in SNS vermeintlich beeinflussen. Die überwiegende Zahl der Forschungsergebnisse basiert auf der Annahme, dass Machine Learning-Algorithmen Social Bots identifizieren (z.B. Varol 2017) oder dass Accounts mit mehr als 50 Tweets pro Tag zuverlässig als Social Bots klassifiziert werden können (Howard/Kollanyi 2016). Beide Annahmen haben sich zwischenzeitlich als zu optimistisch herausgestellt. Dies liegt einerseits an sich stetig wandelnden Taktiken versteckter, koordinierter Social-Media-Kampagnen, dem sogenannten "Astroturfing (Kovic et al. 2018), die die Erkennung über vermeintlich langfristig stabile Eigenschaften oder Signale hinfällig macht, andererseits an der mangelnden Datenbasis, die Forscher ohne privilegierten Zugriff auf Daten von Onlineplattformen zwingt, ihre Analysen auf nur einem Bruchteil der potentiell relevanten Signale durchzuführen. Zusätzlich haben sich entsprechende Studien bisher zu wenig für die Validierung der automatisch von ihnen als Bots klassifizierten Accounts interessiert, so dass entsprechende Ergebnisse über die Reich-

3 Mittlerweile hat Twitter eine Liste der IRA-Accounts und Beteiligter weiterer versteckter Informationskampagnen veröffentlicht. 
weite von Social Bots in der politischen Kommunikation mit Vorsicht zu bewerten sind (Gallwitz/Kreil 2019; Rauchfleisch/Kaiser 2019).

Um die erheblichen Unsicherheiten von Detektionsverfahren $\mathrm{zu}$ umgehen, stützen sich jüngere Arbeiten auf externe Informationen wie z.B. die von Twitter veröffentlichten IRA-Accounts (Lukito et al. 2018), Leaks aus dem chinesischen Regierungsapparat (King et al. 2017) oder Gerichtsdokumente, die Accounts einer verdeckten Social Media-Operation des südkoreanischen Geheimdienstes nennen (Keller et al. 2017, 2019, i.E.). Diese Studien lassen präzisere Aussagen über den Charakter und die Rezeption von versteckten Informationskampagnen $\mathrm{zu}$, als dies mit Verfahren der Bot-Detektion möglich ist. Schließlich zeigen sich dank externer Informationen zu beteiligten Accounts auffällige Koordinationsmuster, auf deren Basis Methoden für die Aufdeckung von Astroturfing generell entwickelt werden können (Keller et al. 2019, i.E.).

\section{Echokammern und Filterblasen}

Ein weiterer Themenschwerpunkt liegt auf der Frage, ob digitale Medien über sogenannte Echokammern (Sunstein 2007) oder Filterblasen (Pariser 2011) zu politischer Polarisierung beitragen. Die Vorstellung von Echokammern basiert darauf, dass Menschen im Falle der Wahl zwischen Informationsquellen immer diejenige wählen würden, von der sie eine Bestärkung ihrer eigenen Meinung erwarten. Diese Annahme wird häufig auf verkürzte Lesarten der psychologischen Ansätze kognitive Dissonanz und Selective Exposure (Zillich 2019) gestützt. Da das frühe Internet von einem Informationsüberfluss geprägt war, gingen manche Autoren davon aus, dass Nutzer sich über die Zeit ausschließlich in Echokammern bewegen würden, die ihre eigenen Meinungen spiegelten. Die Filterblase stellt eine Aktualisierung dieser Idee dar. In einem Internet, das von Plattformen und Apps bestimmt sei, habe der Nutzer gar keine Möglichkeit mehr zur Informationsauswahl. Algorithmisch erstellte Filterblasen spielten, auf Basis personalisierter Profile, Nutzern nur noch Informationen zu, die ihre Meinung stützen, ohne alternative Informationen zur Wahl zu stellen.

Die Ideen der Echokammern und Filterblasen sind sehr erfolgreich und prominent rezipiert. Allerdings berücksichtigen sie schon konzeptionell eine Reihe von Einflussfaktoren nicht, die ihr Auftreten, oder zumindest ihre dominierende Wirkung, einschränken. Zum einen zeigen die Kommunikationsforschung und die Psychologie bereits seit Jahren, dass die Wahl meinungsverstärkender Quellen oder Informationen stark kontextabhängig ist (Kunda 1990), so dass Nutzer nicht automatisch Kommunikation in politisch homogenen Gruppen bevorzugen. Zusätzlich zeigt die Medienrepertoireforschung (Hasebrink/Domeyer 2012), dass gerade politisch interessierte Menschen nicht nur einzelne Medien konsumieren, sondern breite Kombinationen von Medien. Dies führt empirisch nicht zu aus- 
schließlich politisch homogener Mediennutzung, sondern zur Nutzung von Medien aus diversen politischen Lagern (Fletcher/Nielsen 2017; Webster 2014). Dies lässt das Auftreten der in den Konzepten Echokammer und Filterblase beschriebenen Mechanismen zumindest zweifelhaft erscheinen.

Die Analyse von Kommunikations- und Interaktionsdaten von SNS-Nutzern zeigte sowohl Evidenz für Kommunikation in homogenen Gruppen (An et al. 2019) als auch polarisierte Interaktionsnetzwerke (Barberá et al. 2015a; Conover et al. 2011; Lietz et al. 2014). Wir sollten allerdings vorsichtig sein, diese Befunde vorschnell als Bestätigung von Echokammern und Filterblasen zu sehen. Ein zentraler Defekt beider Konzepte ist die deterministische Verknüpfung von empirischen Beobachtungen mit weitreichenden gesellschaftlichen Effekten, ohne diese Verbindung glaubwürdig zu testen. Das Auftreten homogener Kommunikations- oder Interaktionsnetzwerke allein ist kein Beleg für die von Echokammern oder Filterblasen angenommene politische Polarisierung, vor allem da Nutzer nicht ausschließlich in diesen Strukturen kommunizieren (An et al. 2019; Conover et al. 2011).

Jüngere Studien stellen sogar die demokratietheoretischen Grundannahmen der Echokammer-Hypothese in Frage. So zeigen Bail et al. (2018) in einem innovativen Feldexperiment auf Twitter, dass die Rezeption von politisch dissonanten Inhalten nicht etwa zu weniger Polarisierung, sondern zu einer Verhärtung bestehender Einstellungen führt. Die Ergebnisse korrespondieren mit laborexperimentellen Studien, die einen sog. »Backfire Effect« als Folge von dissonanter Informationsaufnahme identifizieren konnten (Nyhan/Reifler 2010).

Schon dieser kurze Überblick zeigt, dass digitale Verhaltensdaten und Methoden aus der Computational Social Science in der Forschung zur politischen Kommunikation geholfen haben, neue Gegenstandsbereiche zu erschließen und etablierte Forschungsfelder weiterzuentwickeln. Allerdings zeigten sich auch bereits Limitationen. Im folgenden Abschnitt skizzieren wir auf dieser Basis eine Forschungsagenda für die sozialwissenschaftliche Integration der CSS in der politischen Kommunikationsforschung.

\section{Forschungsagenda}

Frühen, geradezu euphorischen Erwartungen an das Potential digitaler Verhaltensdaten stehen erhebliche Herausforderungen gegenüber, die zunehmend deutlich werden und die Akzeptanz der CSS in etablierten sozialwissenschaftlichen Kernbereichen einschränken. 


\section{Theorieanbindung}

Die erste Phase der CSS war überwiegend durch deskriptive Studien geprägt, die anhand neuer Methoden und Datensätze Nutzer- oder Elitenverhalten darstellten. In diesen Studien fand selten eine explizite Verortung in Theorien der Sozialwissenschaft statt. Stattdessen wurde häufig auf tagesaktuelle Ängste, in Bezug auf vermeintliche Wirkungen digitaler Medien auf politische Kommunikation, Bezug genommen. Während dieses Vorgehen öffentliche Aufmerksamkeit garantierte, zeigt sich über Zeit, dass diese Arbeitsweise nicht zur Einbindung der CSS im zentralen sozialwissenschaftlichen Diskurs geführt hat. Ohne ernstzunehmende Auseinandersetzung mit sozialwissenschaftlichen Konzepten oder sorgfältige Theoriebildung für neue Kommunikations- oder Interaktionsumgebungen droht sich die CSS in eine Spielart der angewandten Informatik, mit nur schwacher Rezeption und Anknüpfung an die Sozialwissenschaft, zu entwickeln (Jungherr 2019; Jungherr/Theocharis 2017).

Augenblicklich liegt der Schwerpunkt der überwiegenden Zahl an CSS-Studien auf der Anwendung und Entwicklung neuer Methoden oder Datensätze. Hierbei werden allerdings häufig Fragen der Konzeptualisierung und Operationalisierung übersprungen. Ein Beispiel hierfür bieten Arbeiten, die Polarisierung im politischen Diskurs online zeigen wollen (Jungherr 2019). Politische Polarisierung ist ein Phänomen, das seinen Ursprung in den spezifischen Bedingungen US-amerikanischer Politik hat, das unterschiedlich konzeptualisiert wurde (Prior 2013) und das selbst in den USA empirisch überwiegend für politische Eliten und Aktivisten gezeigt werden konnte (Fiorina et al. 2005). Diese Debatte findet sich in den prominentesten Studien der CSS zu diesem Thema nicht wieder. Stattdessen wird der vermeintliche Fakt breiter gesellschaftlicher Polarisierung als Motivation genommen, willkürlich gewählte Interaktionsmuster in digitalen Verhaltensdaten auf homogene oder heterogene Interaktionsmuster hin $\mathrm{zu}$ untersuchen. Werden überwiegend homogene Cluster gefunden, gilt dies als Beleg für Polarisierung (Adamic/Glance 2005; Conover et al. 2011). Es bleibt bei diesen Studien unklar, auf welche Konzeptualisierung politischer Polarisierung sich ihre Befunde beziehen. Sprechen überwiegend homogene Link-Netzwerke zwischen politischen Blogs (Adamic/Glance 2005) oder Retweet-Netzwerke (Conover et al. 2011) tatsächlich für politische Polarisierung? Sind für diese spezifischen Formen von (Teil-)Öffentlichkeiten heterogene Interaktionen überhaupt theoretisch $\mathrm{zu}$ erwarten? Und erlauben es die entsprechenden Befunde tatsächlich, von politischer Polarisierung in Gesellschaften zu sprechen? Um hierzu Antwort zu geben, braucht die CSS eine deutlich stärker ausgeprägte Kultur der bewussten Konzeptualisierung und Operationalisierung.

Parallel hierzu gilt es auch neue Konzepte zu entwickeln, die den veränderten Rahmenbedingungen und Wirkungsweisen zeitgenössischer politischer Kom- 
munikation gerecht werden (Neuman 2016). Die Sozialwissenschaften bieten zahlreiche hilfreiche Konzepte für die Arbeit zu digitaler Kommunikation. Dennoch bedürfen einige davon zumindest der Anpassung, wenn nicht sogar dem Ersatz, um Phänomenen digitaler Kommunikation gerecht zu werden. Seien es diskursive Macht in der neuen Konstellation von Mediensystemen (Jungherr et al. 2019), neue Erwartungen an Nachrichtennutzungsverhalten (de Zuñiga et al. 2017) oder neue Formen der politischen Partizipation (van Deth/Theocharis 2018); neue Phänomene brauchen neue Konzepte, um die Potentiale der CSS nutzbar zu machen.

\section{Datenerhebungsverfahren und Rezeptionsmessung}

Zusätzlich gilt es auch, Fragen der Datenerhebung und der Messung gesellschaftlicher Phänomene kritischer zu reflektieren. Wichtig ist hier die Limitation großer Teile der bestehenden CSS-Forschung in der Nutzung digitaler Spurendaten. Diese Daten stellen Beobachtungen des Verhaltens von Nutzern bestimmter Onlineplattformen dar. Wir haben es allerdings mit einer Stichprobe zu tun, die zweifach auf Selbst-Selektion basiert: Politikwissenschaftlich relevante, digitale Verhaltensdaten werden nur von denjenigen Nutzern generiert, die aktive SNS-Nutzer sind (Mellon/Prosser 2017) und unter dieser spezifischen Population von einer Gruppe an Nutzern, die ein hinreichendes politisches Interesse aufweisen, um öffentlich sichtbar Inhalte $\mathrm{zu}$ »liken«, »sharen« oder sogar eigene Beiträge auf Facebook oder Twitter zu erstellen. Dies ist solange kein Problem, insofern sich die Studie nur auf politisches Verhalten auf den jeweiligen Onlineplattformen beschränkt. Dies erlaubt jedoch keine Rückschlüsse auf die Gesamtbevölkerung oder politisches Verhalten im Allgemeinen.

Erschwert wird die Problematik der Zusammensetzung der Stichprobe noch zusätzlich dadurch, dass digitale Verhaltensdaten zwar reiche Informationen über öffentliches Online-Verhalten enthalten, aber keine Daten über sozio-demographische Eigenschaften der Nutzer oder das Verhalten außerhalb der Online-Plattform. Dies macht es schwierig, die tatsächliche Verzerrung des Samples zu identifizieren oder die Messung gesellschaftlicher Phänomene in Spurendaten mit anderen Messungsarten zu validieren (Jungherr et al. 2016, 2017).

Es gibt allerdings neue Forschungsdesigns, die es ermöglichen, digitale Verhaltensdaten für Rezeptions- und Wirkungsmessungen heranzuziehen. So erlauben es Feldexperimente innerhalb von SNS, Effekte von Medienrezeption auf politische Einstellungen zu schätzen - unter experimenteller Kontrolle auf alternative Medienstimuli in SNS sowie sich wandelnde Plattformcharakteristika (Bail et al. 2018; Theocharis/Lowe 2015).

Eine weitere Möglichkeit, digitale Verhaltensdaten mit der individuellen Ebene $\mathrm{zu}$ verbinden ist ihre Verlinkung mit Umfragen (Stier et al. 2019). So können Facebook- oder Twitter-Nutzer befragt werden (Jäger 2017; Vaccari et al. 2015b) 
oder aber Befragte in Online- oder bevölkerungsrepräsentativen Umfragen um ihre informierte Zustimmung zur Sammlung ihrer digitalen Verhaltensdaten gebeten werden. Derartige verlinkte Daten haben bereits dazu beigetragen, Verzerrungen in Selbsteinschätzungen im Medienkonsum zu schätzen (Guess 2015; Scharkow 2016) und substantielle Fragestellungen aus der politischen Kommunikation wie z.B. die Rezeption politischer Nachrichten in Facebook zu untersuchen (Wells/Thorson 2017).

Schließlich ist der Forscher bei der Erhebung von digitalen Verhaltensdaten, z.B. über die Programmierschnittstellen von SNS, darauf angewiesen, dass die Plattformen qualitativ hochwertige und repräsentative Daten zur Verfügung stellen. Im Falle der Twitter-Programmierschnittstellen (APIs) gibt es erhebliche Verzerrungen, die allerdings nur schwer zu rekonstruieren und nachvollziehbar sind (Morstatter et al. 2013). Noch schwerwiegender sind generelle Änderungen von $\mathrm{Zu}$ griffsrechten wie z.B. Facebooks Deaktivierung der Graph API, wonach der Wissenschaft sogar der Zugriff auf öffentliche Facebook-Daten verwehrt bleibt. Forscher rufen bereits dazu auf, Daten aus sozialen Netzwerken unter Umgehung der APIs zu erheben (Freelon 2018). Eine rechtlich und ethisch unverfänglichere Variante zur Datenerhebung ist die Datenspende. Seit Inkrafttreten der europäischen Datenschutz-Grundverordnung müssen Online-Plattformen den Nutzern ihre persönlichen Daten gebündelt und übersichtlich zum Download anbieten (Datenportabilität, Art. 20 EU-DSGVO). Diese Daten könnten dann unter Anwendung angemessener Pseudonymisierungs- und Datenschutzmaßnahmen von den Nutzern für wissenschaftliche Zwecke zur Verfügung gestellt werden.

Wie bei der Verlinkung von Umfrage- und digitalen Verhaltensdaten ist die informierte Einwilligung und Kooperationsbereitschaft von Teilnehmern auch bei der Datenspende essentiell. Daher stehen Zugewinnen in der wissenschaftlichen Kontrolle, der internen Validität und dem Wissen über die Nutzerpopulation Einbußen in der Skalierbarkeit der Datenerhebung gegenüber. Es benötigt noch einiges an Grundlagenforschung und innovativen Rekrutierungsmethoden, um qualitativ hochwertige Stichproben von Bürgern mit digitalen Verhaltensdaten anzureichern (Stier et al. 2019).

\section{Institutionelle Verankerung}

Die weitere Etablierung der CSS in den Sozialwissenschaften steht auch vor spezifischen institutionellen Herausforderungen. Zum einen erfordert der stetige technologische Wandel und die damit verbundene Steigerung an Datenquellen, -mengen und Analysemethoden zunehmend vielfältige Methodenkenntnisse und Ressourcenzugriffe, die immer seltener von einzelnen Forschern zu bewältigen sind. Dies legt die Nutzung eines in den Naturwissenschaften und der Informatik verbreiteten Lab-Ansatzes nahe, der große Forscherteams mit vielfältigen Spezia- 
lisierungen zusammenbringt. Vor dem Hintergrund verfügbarer Personalmittel stellt dies die meisten sozialwissenschaftlichen Institute, von einzelnen Professuren ganz zu schweigen, vor eine erhebliche Herausforderung. Dies macht die Zusammenarbeit mit Informatikern zu einer Vorbedingung langfristig erfolgreicher CSS. Direkt damit verbunden sind Fragen der Finanzierung entsprechender Forschungsverbünde. Während Interdisziplinarität ein in der Wissenschaft breit geteiltes Ziel ist, orientieren sich Publikationsprozesse, Forschungsfinanzierung und Berufungen noch immer vorwiegend an fachspezifischen Grenzen. Dies stellt eine entscheidende Hürde für die nachhaltige Etablierung der CSS in der Sozialwissenschaft dar.

Die Breite an Aufgaben und Methoden der CSS hat auch Konsequenzen für die sozialwissenschaftliche Methodenausbildung. Während es wünschenswert wäre, die CSS in die Methodenausbildung mit aufzunehmen, stellt sich dies praktisch als schwierig heraus. Letztlich geht es um vieldimensionale Aspekte der Datensammlung, -verarbeitung, -aufbereitung und -analyse. Auch die Datenanalyse fächert sich in unterschiedliche Teilbereiche auf: Machine Learning, Netzwerkanalyse, quantitative Textanalyse oder Bildanalysen sind nur einige Beispiele für prominente, in der CSS verwendete, Analysemethoden. Nur wenige sozialwissenschaftliche Institute werden dieses vielfältige Methodenset in ihrer Ausbildung allein abbilden können. Interdisziplinär unterrichtete Masterprogramme bieten hier vielversprechende Alternativen, um die CSS gezielt in der sozialwissenschaftlichen Ausbildung zu verankern.

\section{Fazit}

Die Computational Social Science bietet ein vielversprechendes neues Instrumentarium an Datentypen und Analysemethoden für die politische Kommunikationsforschung. Ihr Potential erstreckt sich von der Arbeit zu neuen Fragestellungen und Phänomenen digitaler Kommunikation hin zu neuen Analysemöglichkeiten für traditionelle Fragen des Feldes. Um dieses Potential freizusetzen, gilt es jedoch für die CSS und ihre Praktiker, einige Anfangsschwierigkeiten zu überwinden. Gerade sozialwissenschaftlich ausgebildete Forscher sollten ihre Kernkompetenzen nicht methoden-, software-, und datenfixierten Zielen unterordnen. Es gilt, in der CSS und der Anwendung ihrer Methoden gezielt und systematisch Fragen der Theoriebildung, Konzeptualisierung, Datenqualität und Messung zu betonen, um so anschlussfähig für Debatten in der Sozialwissenschaft zu bleiben. Darüber hinaus sind auch forschungspraktische Fragen zu adressieren. Wie entwickeln wir nachhaltig erfolgreiche interdisziplinäre Forschungspartnerschaften, die institutionell verankert sind? Und wie stellen wir die Finanzierung entsprechender Forschungsprojekte in streng disziplinär organisierten Forschungsförde- 
rungsstrukturen sicher? Die Antwort auf diese Fragen wird darüber entscheiden, ob das Forschungspotential der CSS in der Sozialwissenschaft realisierbar ist.

\section{Literaturverzeichnis}

Adamic, Lada A./Glance, Natalie (2005): The political blogosphere and the 2004 US election: Divided they blog. In: Proceedings of the Third International Workshop on Link Discovery, S. 36-43.

Allcott, Hunt/Gentzkow, Matthew (2017): Social Media and Fake News in the 2016 Election. In: Journal of Economic Perspectives 31 (2), S. 211-236.

An, Jisun/Kwak, Haewoon/Posegga, Oliver/Jungherr, Andreas (2019): Political Discussions in Homogeneous and Cross-Cutting Communication Spaces. In: Proceedings of the Thirteenth International Conference on Web and Social Media, S. 68-79.

Arzheimer, Kai (2015): The AfD: Finally a Successful Right-Wing Populist Eurosceptic Party for Germany? In: West European Politics 38 (3), S. 535-556.

Bail, Christopher A. et al. (2018): Exposure to opposing views on social media can increase political polarization. In: PNAS 115 (37), S. 9216-9221.

Barberá, Pablo et al. (2015a): Tweeting from left to right: Is online political communication more than an echo chamber? In: Psychological Science 26 (10), S. 1531-1542.

Barberá, Pablo et al. (2015b): The Critical Periphery in the Growth of Social Protests. In: PLoS ONE 10 (11).

Bennett, W. Lance/Segerberg, Alexandra (2013): The logic of connective action. Digital media and the personalization of contentious politics. New York.

Bennett, W. Lance/Segerberg, Alexandra/Yang, Yunkang (2018): The Strength of Peripheral Networks: Negotiating Attention and Meaning in Complex Media Ecologies. In: Journal of Communication 68 (4), S. 659-684.

Borucki, Isabelle (2019): Politische Parteien zwischen Sein oder nicht Sein? Digitale Transformation als Organisationsumbruch. In: Hofman et al. (Hg.): Politik in der digitalen Gesellschaft. Bielefeld, S. 123-150.

Chadwick, Andrew (2013): The hybrid media system. Politics and power. Oxford.

Conover, Michael et al. (2011): Political Polarization on Twitter. In: Proceedings of the Fifth International AAAI Conference on Web and Social Media, S. 89-96.

De Zúñiga, Homero Gil/Weeks, Brian/Ardèvol-Abreu, Alberto (2017): Effects of the News-Finds-Me Perception in Communication. In: Journal of ComputerMediated Communication 22 (3), S. 105-123.

Dubois, Elizabeth/Gaffney, Devin (2014): The Multiple Facets of Influence: Identifying Political Influentials and Opinion Leaders on Twitter. In: American Behavioral Scientist 58 (10), S. 1260-1277. 
Entman, Robert M./Usher, Nikki (2018): Framing in a Fractured Democracy: Impacts of Digital Technology on Ideology, Power and Cascading Network Activation. In: Journal of Communication 68 (2), S. 298-308.

Ernst, Nicole et al. (2017): Extreme parties and populism. An analysis of Facebook and Twitter across six countries. In: Information, Communication \& Society 20 (9), S. 1347-1364.

Fiorina, Morris P./Abrams, Samuel J./Pope, Jeremy C. (2005): Culture war: The myth of a polarized America. New York.

Fitzpatrick, Jasmin (2019): Potenziale sozialer Medien zwischen Wunsch und Wirklichkeit - Welche Chancen bieten soziale Medien für politischer Akteure und ihre Erforschung? In: Hofman et al. (Hg.): Politik in der digitalen Gesellschaft. Bielefeld, S. 171-186.

Fletcher, Richard/Kleis Nielsen, Rasmus (2017): Are News Audiences Increasingly Fragmented? A Cross-National Comparative Analysis of Cross-Platform News Audience Fragmentation and Duplication. In: Journal of Communication 67 (4), S. 476-498.

Freelon, Deen (2018): Computational Research in the Post-API Age. In: Political Communication 35 (4), S. 665-668.

Freelon, Deen/Karpf, David (2015): Of big birds and bayonets: hybrid Twitter interactivity in the 2012 Presidential debates. In: Information, Communication \& Society 18 (4), S. 390-406.

Gallwitz, Florian/Kreil, Michael (2019): Die Mär von `Social Bots‘. In Tagesspiegel. URL: https://background.tagesspiegel.de/die-maer-von-social-bots (03.07.2019).

Grinberg, Nir et al. (2019): Fake news on Twitter during the 2016 U.S. presidential election. In: Science 363 (6425), S. 374-378.

Guess, Andrew. M. (2015): Measure for Measure. An Experimental Test of Online Political Media Exposure. In: Political Analysis 23 (1), S. 59-75.

Guess, Andrew. M./Nyhan, Brendan/Reifler, Jason (2018): Selective Exposure to Misinformation: Evidence from the consumption of fake news during the 2016 US presidential campaign. Dartmouth College. URL: https://www.dartmouth. edu/ nyhan/fake-news-2016.pdf (10.03.2018).

Hasebrink, Uwe/Domeyer, Hanna (2012): Media repertoires as patterns of behaviour and as meaningful practices: A multimethod approach to media use in converging media environments. Participations 9 (2), S. 757-779.

Howard, Philip N./Kollanyi, Bence (2016): Bots, \#StrongerIn, and \#Brexit: Computational Propaganda during the UK-EU Referendum. URL: https://arxiv.org/ pdf/1606.06356.pdf (03.04.2019).

Howison, James/Wiggins, Andrea/Crowston, Kevin (2011): Validity Issues in the Use of Social Network Analysis with Digital Trace Data. In: Journal of the Association for Information Systems 12 (12), S. 767-797. 
Iyengar, Shanto/Hahn, Kyu S. (2009): Red media, blue media. Evidence of ideological selectivity in media use. In: Journal of Communication 59 (1), S. 19-39.

Jäger, Kai (2017): The potential of online sampling for studying political activists around the world and across time. In: Political Analysis 25 (3), S. 329-343.

Jungherr, Andreas (2014): The logic of political coverage on Twitter: Temporal dynamics and content. In: Journal of Communication 64 (2), S. 239-259.

Jungherr, Andreas (2015): Analyzing Political Communication with Digital Trace Data. Basel.

Jungherr, Andreas (2016): Twitter use in election campaigns: A systematic literature review. In: Journal of Information Technology \& Politics 13 (1), S. 72-91.

Jungherr, Andreas (2019): Normalizing digital trace data. In: Stroud/McGregor (Hg.): Digital Discussions. New York, S. 9-35.

Jungherr, Andreas/Jürgens, Pascal/Schoen, Harald (2012): Why the Pirate Party Won the German Election of 2009 or the Trouble with Predictions. In: Social Science Computer Review 30 (2), S. 229-234.

Jungherr, Andreas/Posegga, Oliver/An, Jisun (2019): Discursive Power in Contemporary Media Systems: A Comparative Framework. In: The International Journal of Press/Politics. DOI: https://doi.org/10.1177/1940161219841543.

Jungherr, Andreas/Schoen, Harald/Jürgens, Pascal (2016): The Mediation of Politics through Twitter. An Analysis of Messages posted during the Campaign for the German Federal Election 2013. In: Journal of Computer-Mediated Communication 21 (1), S. 50-68.

Jungherr, Andreas et al. (2017): Digital Trace Data in the Study of Public Opinion: An Indicator of Attention Toward Politics Rather Than Political Support. In: Social Science Computer Review 35 (3), S. 336-356.

Jungherr, Andreas/Theocharis, Yannis (2017): The empiricist's challenge: Asking meaningful questions in political science in the age of big data. In Journal of Information Technology\& Politics 14 (2), S. 97-109.

Jürgens, Pascal/Jungherr, Andreas/Schoen, Harald (2011): Small Worlds with a Difference. New Gatekeepers and the Filtering of Political Information on Twitter. In: Proceedings of the Third International Web Science Conference, S. 21-25.

Keller, Franziska/Schoch, David/Stier, Sebastian/Yang, JungHwan (2017): How to Manipulate Social Media: Analyzing Political Astroturfing Using Ground Truth Data from South Korea. In: Proceedings of the Eleventh International AAAI Conference on Web and Social Media, S. 564-567.

Keller, Franziska et al. (2019, in Erscheinung): Political Astroturfing on Twitter: How to Coordinate a Disinformation Campaign. In: Political Communication.

King, Gary/Pan, Jennifer/Roberts, Margaret E. (2017): How the Chinese government fabricates social media posts for strategic distraction, not engaged argument. In: American Political Science Review 111 (3), S. 484-501. 
Kovic, Marko et al. (2018): Digital Astroturfing in Politics: Definition, Typology, and Countermeasures. In Studies in Communication Sciences 18 (1), S. 69-85.

Kunda, Ziva (1990): The case for motivated reasoning. Psychological Bulletin 108 (3), S. 480-498.

Lazer, David et al. (2009): Computational social science. In: Science 323 (5915), S. 721-723.

Lietz, Haiko et al. (2014): When Politicians Talk. Assessing Online Conversational Practices of Political Parties on Twitter. In: Proceedings of the Eighth International AAAI Conference on Weblogs and Social Media, S. 285-294.

Lukito, Josephine et al. (2018): The Twitter Exploit: How Russian Propaganda Infiltrated U.S. News. URL: https://uwmadison.app.box.com/v/TwitterExploit (20.03.2018).

Mellon, Jonathan/Prosser, Christopher (2017): Twitter and Facebook are not representative of the general population. Political attitudes and demographics of British social media users. In: Research \& Politics 4 (3).

Messing, Solomon/Westwood, Sean J. (2014): Selective Exposure in the Age of Social Media. Endorsements Trump Partisan Source Affiliation When Selecting News Online. In: Communication Research 41 (8), S. 1042-1063.

Morstatter, Fred et al. (2013): Is the Sample Good Enough? Comparing Data from Twitter's Streaming API with Twitter's Firehose. In: Proceedings of the Seventh International AAAI Conference on Weblogs and Social Media, S. 400-408.

Neuman, Russell W. (2016): The digital difference. Harvard, Massachusetts.

Neuman, Russell W. (2014): The Dynamics of Public Attention: Agenda-Setting Theory Meets Big Data. In: Journal of Communication 64 (2), S. 193-214.

Newman, Nic et al. (2018): Reuters Institute Digital News Report 2018. URL: http://media.digitalnewsreport.org/wp-content/uploads/2018/06/digitalnews-report-2018.pdf (07.05.2019).

Nielsen, Rasmus Kleis/Vaccari, Cristian (2013): Do people »like« politicians on Facebook? Not really. Large-Scale Direct Candidate-to-Voter Online Communication as an Outlier Phenomenon. In: International Journal of Communication 7 (7), S. 2333-2356.

Nuernbergk, Christian (2016): Political Journalists' Interaction Networks. In: Journalism Practice 10 (7), S. 868-879.

Nyhan, Brendan/Reifler, Jason (2010): When Corrections Fail: The Persistence of Political Misperceptions. In: Political Behavior 32 (2), S. 303-330.

Pariser, Eli (2011): The filter bubble. What the Internet is hiding from you. New York.

Pennycook, Gordon/Cannon, Tyrone D./Rand, David G. (2018): Prior exposure increases perceived accuracy of fake news. In: Journal of Experimental Psychology. 147 (12), S. 1865-1880. 
Posegga, Oliver/Jungherr, Andreas (2019): Characterizing Political Talk on Twitter: A Comparison Between Public Agenda, Media Agendas, and the Twitter Agenda with Regard to Topics and Dynamics. In: Proceedings of the 52nd Hawaii International Conference on System Sciences, S. 2590-2599.

Prior, Markus (2013): Media and Political Polarization. In: Annual Review of Political Science 16 (1), S. 101-127.

Quinlan, Stephen et al. (2017): >Show me the money and the party! - Variation in Facebook and Twitter adoption by politicians. In: Information, Communication \& Society 21 (8), S. 1031-1049.

Rauchfleisch, Adrian/Kaiser, Jonas (2019): The False Positive Problem of Automatic Bot Detection. AoIR 2019: The 2oth Annual Conference of the Association of Internet Researchers.

Scharkow, Michael (2016): The Accuracy of Self-Reported Internet Use? A Validation Study Using Client Log Data. In: Communication Methods and Measures 10 (1), S. 13-27.

Steinert-Threlkeld, Zachary C. (2017): Spontaneous collective action. Peripheral mobilization during the Arab Spring. In: American Political Science Review 111 (2), S. $379-403$.

Stier, Sebastian et al. (2018a): Election Campaigning on Social Media: Politicians, Audiences and the Mediation of Political Communication on Facebook and Twitter. In: Political Communication 35 (1), S. 50-74.

Stier, Sebastian et al. (2019): Integrating Survey Data and Digital Trace Data. Key Issues in Developing an Emerging Field. In: Social Science Computer Review. DOI: https://doi.org/10.1177/0894439319843669.

Stier, Sebastian et al. (2017): When populists become popular: comparing Facebook use by the right-wing movement Pegida and German political parties. In: Information, Communication \& Society 20 (9), S. 1365-1388.

Stier, Sebastian/Schünemann, Wolf J./Steiger, Stefan (2018b): Of activists and gatekeepers. Temporal and structural properties of policy networks on Twitter. In: New Media \& Society 20 (5), S. 1910-1930.

Sunstein, Cass R. (2007): Republic.com 2.0: Princeton, NJ.

Theocharis, Yannis/Lowe, Will (2015): Does Facebook increase political participation? Evidence from a field experiment. In: Information, Communication \& Society 19 (10), S. 1465-1486.

Trilling, Damian (2015): Two Different Debates? Investigating the Relationship Between a Political Debate on TV and Simultaneous Comments on Twitter. In: Social Science Computer Review 33 (3), S. 259-276.

Tumasjan, Andranik et al. (2011): Election Forecasts with Twitter: How 140 Characters Reflect the Political Landscape. In: Social Science Computer Review 29 (4), S. $402-418$. 
Vaccari, Cristian/Chadwick, Andrew/O'Loughlin, Ben (2015a): Dual Screening the Political: Media Events, Social Media, and Citizen Engagement. In: Journal of Communication 65 (6), S. 1041-1061.

Vaccari, Cristian et al. (2015b): Political Expression and Action on Social Media. Exploring the Relationship Between Lower- and Higher-Threshold Political Activities Among Twitter Users in Italy. In: Journal of Computer-Mediated Communication 20 (2), S. 221-239.

Van Deth, Jan W./Theocharis, Yannis (2017): Political participation in a changing world: Conceptual and empirical challenges in the study of citizen engagement. New York.

Vergeer, Maurice/Hermans, Liesbeth/Sams, Steven (2013): Online social networks and micro-blogging in political campaigning: The exploration of a new campaign tool and a new campaign style. In: Party Politics 19 (3), S. 477-501.

Vosoughi, Soroush/Roy, Deb/Aral, Sinan (2018): The spread of true and false news online. In: Science 359 (6380), S. 1146-1151.

Wardle, Claire/Derakhshan, Hossein (2017): Information Disorder: Toward an interdisciplinary framework for research and policymaking. In: Council of Europe Report DGI (2017)09. URL: https://rm.coe.int/information-disordertoward-an-interdisciplinary-framework-for-researc/168076277c (05.05.2019).

Webster, James G, 2014: The marketplace of attention: How audiences take shape in a digital age. Cambridge, Massachusetts.

Wells, Chris et al. (2016): How Trump Drove Coverage to the Nomination. Hybrid Media Campaigning. In: Political Communication 33 (4), S. 669-676.

Wells, Chris/Thorson, Kjerstin (2017): Combining Big Data and Survey Techniques to Model Effects of Political Content Flows in Facebook. In: Social Science Computer Review 35 (1), S. 33-52.

Yang, JungHwan/Kim, Young Mie (2017): Equalization or Normalization? Voter-candidate engagement on Twitter in the 2010 U.S. midterm elections. In: Journal of Information Technology \& Politics 14 (3), S. 232-247.

Zhang, Yini et al. (2019): Whose Lives Matter? Mass Shootings and Social Media Discourses of Sympathy and Policy, 2012-2014. In: Journal of Computer-Mediated Communication. DOI: https://doi.org/10.1093/jcmc/zmzo09.

Zillich, Arne Freya (2019): Konsistenztheorien und Selective Exposure. BadenBaden. 